\title{
Susceptibilidad a antimicrobianos de patógenos respiratorios en niños provenientes de la comunidad
}

\author{
José M. Guevara1 ${ }^{1}$ Rosaluz Aróstegui², Wini Agurto², Iliana Sobrevilla², \\ Esther Valencia ${ }^{1}$, Nazario Silva ${ }^{3}$ \\ ${ }^{1}$ Instituto de Medicina Tropical Daniel A. Carrión - UNMSM. ${ }^{2}$ Otorrinolaringología del Hospital Nacional \\ Docente Madre-Niño San Bartolomé. ${ }^{3}$ Epidemiología del Hospital Nacional Docente Madre-Niño San Bartolomé \\ y Dpto. de Medicina Preventiva y Salud Pública Facultad de Medicina - UNMSM.
}

\begin{abstract}
Resumen
OBJETIVO: Determinar la resistencia de los patógenos respiratorios a diferentes antimicrobianos. MATERIAL Y MÉTODOS: Entre abril y noviembre de 2002 se estudió 177 pacientes que asistieron al consultorio externo de otorrinolaringología del Hospital Nacional Docente Madre-Niño San Bartolomé. RESULTADOS: Streptococcus pneumoniae fue la bacteria patógena más aislada (57,2\%), luego Moraxella catarrhalis (42,7\%), Staphylococcus aureus $(18,6 \%)$ y en pequeña cantidad Haemophilus influenzae $(3,4 \%)$ y Streptococcus pyogenes $(0,7 \%)$. Streptococcus pneumoniae presentó $31,3 \%$ de resistencia a la penicilina. El $96,7 \%$ de Moraxella catarrhalis fueron productoras de betalactamasa y 7,4\% de losStaphylococcus aureus fueron resistentes a la oxacilina. CONCLUSIÓN: Streptococcus pneumoniae es el principal agente causal de los procesos infecciosos altos en niños y su resistencia a la penicilina aumentó a 31,3\%
\end{abstract}

Palabras clave: Streptococcus pneumoniae; resistencia microbiana a las drogas; resistencia a penicilina; enfermedades respiratorias.

\section{Respiratory pathogenic microorganism's sensitivity in community children Summary}

OBJECTIVE: To determine the respiratory pathogens resistance to different antimicrobial drugs. MATERIAL AND METHODS: From April through November 2002, 177 patients attending the Mother-Child National Teaching Hospital Otorhinolaryngology outpatient's office were studied. RESULTS: Streptococcus pneumoniae was the most frequently isolated pathogenic bacteria $(57,2 \%)$, followed byMoraxellacatarrhalis (42,7\%), Staphylococcus aureus (18,6\%), Haemophilus influenzae (3,4\%) and Streptococcus pyogenes $(0,7 \%)$. Streptococcus pneumoniae showed resistance to the penicillin in 31,3\%; 96,7 of Moraxellacatarrhalis were producers of betalactamase and 7,4\% of Staphylococcus aureus showed resistance to oxacillin. CONCLUSION: Streptococcus pneumoniae is the principal causal factor of respiratory infections in children and its resistance to the penicillin increased to $31,3 \%$.

Key words: Streptococcus pneumoniae; drug resistance, microbial; penicillin resistance; respiratory tract diseases.

\section{INTRODUCCIÓN}

Correspondencia:

Dr. José María Guevara Duncan

Instituto de Medicina Tropical Daniel A. Carrión

UNMSM - Departamento de Microbiología.

Jr. José Santos Chocano 199. Urb. San Joaquín

Callao 02, Perú

E-mail: jomaguedu34@hotmail.com 
Las bacterias patógenas respiratorias están incrementando su resistencia a los antimicrobianos en las infecciones adquiridas en la comunidad.

Streptococcus pneumoniae, Haemophilus influenzae y Moraxella catarrhalis constituyen los principales patógenos en las infecciones del tracto respiratorio adquiridas en la comunidad y el más agresivo es el Streptococcus pneumoniae; éste provoca más de un millón de muertes en niños menores de 5 años, cada año, en países en desarrollo y es el principal causante de la neumonía adquirida en la comunidad; además, en los últimos 10 a 20 años ha desarrollado resistencia a la mayor parte de los antibióticos empleados en su tratamiento $\left(^{(}\right)$.

Streptococcus pneumoniae se vuelve resistente a la penicilina por mutaciones en sus proteínas fijadoras de penicilina (PBP) y Haemophilus influenzae y Moraxella catarrhalis adquieren resistencia a la ampicilina por producción de la enzima betalactamasa.

El uso indiscriminado de antibióticos en el tratamiento empírico de infecciones respiratorias en nuestro país se ha convertido en un problema de Salud Pública. Por eso, el conocimiento de la susceptibilidad actual es sumamente importante para que el clínico elija el mejor tratamiento.

\section{MATERIAL Y MÉTODOS}

Entre abril y noviembre del año 2002, se estudió 177 pacientes en el consultorio externo de otorrinolaringología del Hospital Nacional Docente Madre Niño San Bartolomé, quienes presentaron signos de infección respiratoria alta y no habían recibido antibióticos.

Se utilizó un cuestionario como instrumento de medición, que incluía información epidemiológica.

Las muestras fueron obtenidas con hisopos por los especialistas y colocadas en tubos que contenían el medio de transporte Stuart. Los medios de transporte con las muestras fueron recogidos el mismo día y llevados al Instituto de Medicina Tropical
Daniel A. Carrión para su procesamiento.

Se empleó los siguientes medios de cultivo: agar Columbia-sangre de carnero, agar Columbia sangre de carnero con gentamicina ( $)$, agar selectivo para Moraxella catarrhalis $\left({ }^{3}\right)$ y agar manitol salado.

La identificación de Streptococcus pneumoniae se hizo empleando discos de Optochin y en algunos casos dudosos bilis solubilidad. La identificación de Haemophilus influenzae se hizo empleando el satelitismo con estafilococo. La de Moraxella catarrhalis con la prueba de oxidasa, por el metabolismo de carbohidratos y con el agar DNAsa (hidroliza el ADN). La de Staphylococcus aureus se hizo por la coagulasa. La de Streptococcus pyogenes por serología.

La producción de betalactamasa en $H$. influenzae y $M$. catarrhalis se detectó empleando discos de cefalosporina cromógena (Nitrocefina).

Se hizo los antibiogramas empleando el método de disco-difusión estandarizado siguiendo las reglas del NCCLS.

Para el análisis se utilizó el programa estadístico SPSS.

\section{RESULTADOS}

La edad de los 177 pacientes fluctuó entre $0 \mathrm{y}$ 14 años y 105 (94\%) fueron menores de 5 años; $108(61 \%)$ del sexo masculino y 69 (39\%) del femenino (Tabla 1) y la razón hombre/mujer fue 1:5. De 30 distritos, los distritos de mayor procedencia fueron Cercado, Comas y San Martín de Porras.

Los principales síntomas y signos que presentaron fueron secreción purulenta, tupidez nasal y tos productiva (Tabla 2 ).

Se tomó muestras de secreción nasal purulenta o serosa 139 (78,5\%), de inflamación faríngea 30 $(16,9 \%)$ y de secreción ótica $8(4,5 \%)$.

De las 177 muestras, $145(81,9 \%)$ tuvieron alguna bacteria patógena, mientras $32(18,1 \%)$ fueron negativas a gérmenes patógenos cultivables. 
Tabla 1.- Edad y sexo de los niños estudiados.

\begin{tabular}{lrrrr}
\hline \multicolumn{1}{c}{ Sexo } & \multicolumn{3}{c}{ Edad } & Total \\
\cline { 2 - 4 } & $0-4$ & $5-9$ & $10-14$ & \\
\hline Masculino & 64 & 33 & 11 & 108 \\
Femenino & 41 & 18 & 10 & 69 \\
Total & 105 & 51 & 21 & 177 \\
\hline
\end{tabular}

Los cultivos positivos fueron: Streptococcus pneumoniae 83 (57,2\%), Moraxella catarrhalis 62 (42,7\%), Staphylococcus aureus 27 (18,6\%), Haemophilus influenzae 5 (3,4\%), Streptococcus pyogenes 1 (0,7\%), bacilos Gram negativos $14(9,6 \%)$.

La combinación que frecuentemente se presentó en 39 cultivos $(27 \%)$ fue Streptococcus pneumoniae con Moraxella catarrhalis.

Streptococcus pneumoniae se aisló de secreción nasal en 71 pacientes, de secreción faríngea en 10 y de secreción ótica en 2.

Los síntomas y signos que presentaron los pacientes a los que se les aisló Streptococcus pneumoniae fueron rinorrea purulenta, tupidez nasal y tos productiva (Tabla 2 ).

Tabla 2.- Síntomas y signos.

Pacientes estudiados en el Servicio de Otorrinolaringología

\begin{tabular}{lrr} 
& $\mathrm{n}=117$ & \multicolumn{1}{c}{$\%$} \\
\cline { 2 - 3 } Rinorrea purulenta & 148 & 83,6 \\
Rinorrea serosa & 15 & 8,5 \\
Tupidez nasal & 83 & 35,6 \\
Tos productiva & 52 & 29,4 \\
Inflamación faríngea & 49 & 27,7
\end{tabular}

Pacientes a quienes se aisló Streptococcus pneumoniae

Rinorrea purulenta

Rinorrea serosa

Tupidez nasal

Tos productiva

Inflamación faríngea

\begin{tabular}{rr}
$\mathrm{n}=83$ & \multicolumn{1}{c}{$\%$} \\
\hline 77 & 92,0 \\
6 & 7,2 \\
33 & 39,7 \\
25 & 30,0 \\
16 & 19,2
\end{tabular}

En el resultado del antibiograma de las 83 cepas de Streptococcus pneumoniae, cabe destacar que 26 cepas $(31,3 \%)$ fueron resistentes a penicilina, porque no dieron halo de inhibición con el disco de oxacilina y $7(6,4 \%)$ fueron poco sensibles a la penicilina, porque hubo halo de inhibición menor de $20 \mathrm{~mm}$. Además, hubo varios antibióticos que no encontraron resistencia en los neumococos testados (Tabla 3).

En el antibiograma de las cepas de Moraxella catarrhalis, 60 de las 62 cepas fueron productoras de betalactamasa; sin embargo, 31 de éstas (50\%) fueron sensibles a la ampicilina (Tabla 4).

El resultado del antibiograma de las 27 cepas de Staphylococcus aureus, se pueden observar en la Tabla 5, en donde destaca la baja resistencia a la oxacilina, presente en 2 cepas $(7,4 \%)$ y la sensibilidad de $100 \%$ a la vancomicina.

\section{DISCUSIÓN}

Streptococcus pneumoniae, Haemophilus influenzae, Moraxella catarrhalis y Staphylococcus aureus están frecuentemente vinculados con las infecciones agudas respiratorias y en los últimos años se ha observado una gran variación en su resistencia a los antimicrobianos.

En el año $1997\left(^{4}\right)$, reportamos el aislamiento de 36 cepas de Streptococcus pneumoniae procedentes de 80 niños y 20 adultos, 11\% resistentes a la penicilina. Ahora ese porcentaje ha subido a $31,3 \%$, sin considerar que $6,4 \%$ resultó poco sensible y con un mayor número de aislamientos.

Se ha reportado aislamientos de neumococos con mayor resistencia a la penicilina que lo que encontramos nosotros $\left({ }^{5-10}\right)$, así también con menor resistencia $\left({ }^{11-20}\right)$. Ochoa, en $1997\left({ }^{21}\right)$, en niños que acudieron al Hospital Cayetano Heredia de Lima encontró que 5,3\% de Streptococcus pneumoniae eran resistentes a la penicilina.

En el mismo trabajo ( $)$, reportamos el aislamiento de 30 cepas de Moraxella 
Tabla 3. Resultado del antibiograma de 83 cepas de Streptococcus pneumoniae.

\begin{tabular}{lccc}
\hline Antibiótico & Sensible & $\begin{array}{c}\text { Poco sensible } \\
\text { o intermedio }\end{array}$ & Resistente \\
\hline Vancomicina & $83(100, \%)$ & 0 & 0 \\
Rifampicina & $83(100 \%)$ & 0 & 0 \\
Cefotaxima & $82(98,8 \%)$ & $1(1,2 \%)$ & 0 \\
Ceftriaxona & $82(98,8 \%)$ & $1(1,2 \%)$ & 0 \\
Cefuroxima & $81(97,6 \%)$ & $2(2,4 \%)$ & 0 \\
Lincomicina/ & & & \\
Clindamicina & $74(90,2 \%)$ & $1(1,2 \%)$ & $7(8,5 \%)$ \\
Ciprofloxacina & $61(73,5 \%)$ & $14(16,9 \%)$ & $8(9,6 \%)$ \\
Eritromicina & $66(79,5 \%)$ & $4(4,8 \%)$ & $13(15,7 \%)$ \\
Cloranfenicol & $67(80,7 \%)$ & $1(1,2 \%)$ & $15(18,1 \%)$ \\
Tetraciclina & $56(67,5 \%)$ & $4(4,8 \%)$ & $23(27,7 \%)$ \\
Penicilina & $50(60,2 \%)$ & $7(6,4 \%)$ & $26(31,3 \%)$ \\
Cotrimoxazol & $26(31,3 \%)$ & $8(9,6 \%)$ & $49(59,0 \%)$ \\
\hline
\end{tabular}

catarrhalis, de las cuales $70 \%$ eran productoras de betalactamasa. Ahora encontramos que 96,7\% de las cepas la elaboraban, a pesar de que in vitro $50 \%$ de ellas fueron sensibles a la ampicilina.

En la referencia 4 se reporta el aislamiento de Staphylococcus aureus, $15 \%$ resistente a oxacilina, mientras que ahora hay $7,4 \%$ de resistencia.

En el presente trabajo no se pudo tener acceso al E-Test para determinar la concentración mínima inhibitoria a Streptococcus pneumoniae, que era lo ideal.

En lo que respecta a Moraxella catarrhalis, el método de disco-difusión no está estandarizado, por lo que se utilizó la tabla para microorganismos de crecimiento rápido.

En conclusión, Streptococcus pneumoniae es el principal agente causal de las infecciones respiratorias en niños procedentes de la comunidad, ocupando Moraxella catarrhalis y Staphylococcus aureus el segundo y tercer lugar respectivamente. La resistencia a los antibióticos se ha incrementado, en Streptococcus pneumoniae $31,3 \%$ a la penicilina y en Moraxella catarrhalis $96,7 \%$ son productoras
Tabla 4. Resultados del antibiograma de 62 cepas de Moraxella catarrhalis, 60 de las cuales eran productoras de betalactamasa.

\begin{tabular}{lccc}
\hline Antibiotico & Sensible & $\begin{array}{c}\text { Poco sensible } \\
\text { o intermedio }\end{array}$ & Resistente \\
\hline Amoxicilina- & & & \\
Sulbactam & $62(100 \%)$ & 0 & 0 \\
Cefotaxima & $62(100 \%)$ & 0 & 0 \\
Ciprofloxacina & $62(100 \%)$ & 0 & 0 \\
Cloranfenicol & $62(100 \%)$ & 0 & 0 \\
Eritromicina & $57(91,9 \%)$ & $5(8,1 \%)$ & 0 \\
Gentamicina & $62(100 \%)$ & 0 & 0 \\
Rifampicina & $62(100 \%)$ & 0 & 0 \\
Tetraciclina & $60(96,8 \%)$ & $2(3,2 \%)$ & 0 \\
Ceftriaxona & $58(93,5 \%)$ & $3(4,8 \%)$ & $1(1,6 \%)$ \\
Cotrimoxazol & $54(87,1 \%)$ & $4(6,5 \%)$ & $4(6,5 \%)$ \\
Ampicilina & $31(50 \%)$ & $3(4,8 \%)$ & $28(45,2 \%)$ \\
\hline
\end{tabular}

de betalactamasa. Con relación a Staphylococcus aureus, todos fueron sensibles a vancomicina y sólo $7,4 \%$ de resistencia a oxacilina.

\section{AGRADECIMIENTOS}

Al Consejo Superior de Investigación de la UNMSM

Tabla 5. Resultado del antibiograma de 27 cepas de Staphylococcus aureus.

\begin{tabular}{lccc}
\hline Antibiótico & Sensible & Intermedio & Resistente \\
\hline Vancomicina & $27(100 \%)$ & 0 & 0 \\
Rifampicina & $26(96,3 \%)$ & 0 & $1(3,7 \%)$ \\
Gentamicina & $25(92,6 \%)$ & 0 & $2(7,4 \%)$ \\
Oxacilina & $24(88,9 \%)$ & $1(3,7 \%)$ & $2(7,4 \%)$ \\
Cefalotina & $24(88,9 \%)$ & $1(3,7 \%)$ & $2(7,4 \%)$ \\
Lincomicina/ & & & \\
Clindamicina & $21(77,8 \%)$ & $4(14,8 \%)$ & $2(7,4 \%)$ \\
Ciprofloxacina & $21(77,8 \%)$ & $4(14,8 \%)$ & $2(7,4 \%)$ \\
Cloranfenicol & $23(85,2 \%)$ & $1(3,7 \%)$ & $3(11,1 \%)$ \\
Tetraciclina & $19(70,4 \%)$ & $5(18,5 \%)$ & $3(11,1 \%)$ \\
Cotrimoxazol & $22(81,5 \%)$ & 0 & $5(18,5 \%)$ \\
Eritromicina & $9(33,3 \%)$ & $11(40,7 \%)$ & $7(25,9 \%)$ \\
Penicilina & $1(3,7 \%)$ & $1(3,7 \%)$ & $25(92,6 \%)$ \\
\hline
\end{tabular}


por su aporte económico. A la Srta. Sara Paredes por su aporte técnico. Al personal auxiliar del consultorio de Otorrinolaringología del Hospital San Bartolomé por su colaboración.

\section{BIBLIOGRAFÍA}

1. Tauber MG. Pneumonia due to resistant Streptococcus pneumoniae. Schweizerisehe Medizinische Wochenschrift. J Susse Medecine 2000; 130(49): 1873-9.

2. Converse GM, Dillon HC. Epidemiological studies of Streptococcus pneumoniae in Infants. Methods of isolating Pneumococci. J Clin Microbiol 1977; 5(3): 293-6.

3. Vaneechoutte $M$, Verscgraegen $G$, Claeys G, van den Abeele A. Selective medium for Branhamella catarrhalis with acetazolamide as a specific inhibitor of Neisseria spp. J Clin Microbiol 1988; 26(12): 2544-8.

4. Guevara J, Navach J, Aróstegui R, Agurto W, Goytendia A, Chong M, et al. Moraxella catarrhalis en las infecciones respiratorias altas. Anales Fac Med UNMSM 1997; 58(3): 192-8.

5. Tanove S. Clinical and laboratory evaluation of penicillin resistant Streptococcus pneumoniae in relation to the mutations of pbp $1 \mathrm{a}, \mathrm{pbp} 2 \mathrm{~b}$ and $\mathrm{pbp} 2 \mathrm{x}$. Kurume Med J 2001; 48(1): 1-8.

6. Perronec C, Perrone P, Kopetz V, Nedunchezian D, Leggiadro R. Prevalence of penicillin - nonsusceptible pneumococcal bacteremia in a Staten Island community hospital. South Med J 2000; 93(11): 1078-80.

7. Thornsberry C,Salem DF. Resistance in respiratory tract pathogens: an international study $1997-1998$. J Chemother 2000; 12(Suppl 4): 16-20.

8. Schito GC, Debbia EA, Marchese A. The evolving threat of antibiotic resistance in Europe: new data from the Alexander Project. J Antimicrob Chemother 2000; 46(Suppl 1): 3-9.

9. Sader HS, Gales AC, Granacher TD, Pfaller MA, Jones RN. Prevalence of antimicrobial resistance among respiratory tract isolates in Latin America: results from SENTRY antimicrobial survillance program (1997-1998). Braz J Infect Dis 2000; 4(5): 245-54.

10. Baquero F, Garcia-Rodriguez JA, Garcia de Lomas J, Aguilar L. Antimicrobial resistance of 1,113 Streptococcus pneumoniae isolates from patients with respiratory tract infections in Spain: results of a 1 year (1996-1997) multicenter survillance study. Antimicrob Agents Chemother 1999; 43(2): 357-9.

11. Sahin U, Unlu M, Demirci M, Akkaya A, Turgut E.
Penicillin resistance in Streptococcus pneumoniae in Isparta. Respirol 2001; 6(1): 23-6.

12. Thornsberry C, Ogilrie PT, Holley HP Jr, Sahm DF. Survey of susceptibilities of Streptococcus pneumoniae, Haemophilus influenzae, and Moraxella catarrhalis isolates to 26 antimicrobial agents: a prospective U.S. study. Antimicrob Agents Chemother 1999; 43(11): 2612-23.

13. Cornaglia G, Fontana R. Epidemiological survey of bacterial resistance in upper respiratory tract infections in Italy. Intern J Antimicrob Agents 2000; 16(3): 259-62.

14. Fenoll A, Jado I, Vicioso D, Perez A, Casal J.Evolution of Streptococcus pneumoniae serotypes and antibiotc resistance in Spain: update (1990 to 1996). J Clin Microbiol 1998; 36(12): 3447-54.

15. Andrews J, Ashby J, Jerons G, Lines N, Wise R. Antimicrobial resistance in gram-positive pathogens isolated in the UK between October 1996 and January 1997. J Antimicrob Chemother 1999; 45(3): 689-98.

16. Ronchetti MP, Guglielmi F, Latini L, Merolla R, Lorusso G, Bajaksouzian S, et al. Resistance patterns of Streptococcus pneumoniae from children in central Italy. European J Clin Microbiol Infect Dis 1999; 18(5): 376-9.

17. Barry AL. Antimicrobial resistance among clinical isolates of Streptococus pneumoniae in North America. Am J Med 1999; 107(1A): 285-335.

18. Hoban DJ, Karlowsky JA, Zhanel GG. Evolving patterns of resistance to respiratory pathogens in Canada. Canadian Resp J 1999; 6(Suppl A): 27A-30A.

19. Felmingham D, Washington J. Trends in the antimicrobial susceptibility of bacterial respiratory tract pathogens findings of the Alexander Project 1992 - 1996. J Chemother 1999; 11(Suppl 1): 5-21.

20. Felmingham D, Robbins MJ, Tesfaslasie Y, Harding I, Shrimpton S, Gruneberg RN. Antimicrobial susceptibility of community-acquired bewer respiratory tract bacterial pathogens isolated in the UK during the 1995-1996 cold season. J Antimicrob Chemother 1998; 41(3): 411-5.

21. Ochoa TJ.Resistencia de Streptococcus pneumoniae a penicilina en portadores nasofaríngeos menores de 2 años. Tesis para optar el Título de Médico Cirujano, 1997, UPCH. 